\title{
Correction Notice to: Feasibility of precise navigation in high and low latitude regions under scintillation conditions
}

José Miguel Juan ${ }^{1}$, Jaume Sanz ${ }^{1}$, Guillermo González-Casado ${ }^{1}$, Adrià Rovira-Garcia ${ }^{1}$, Adriano Camps $^{1,2}$, Jaume Riba ${ }^{1}$, José Barbosa ${ }^{3}$, Estefania Blanch ${ }^{4}$, David Altadill ${ }^{4}$ and Raul Orus Perez ${ }^{5}$

${ }^{1}$ Universitat Politècnica de Catalunya \& IEEC/CTE-UPC, UPC Campus Nord, 08034 Barcelona, Spain

2 Institut d'Estudis Espacials de Catalunya IEEC/UPC, Edifici Nexus-201, 08034 Barcelona, Spain

${ }^{3}$ RDA - Research and Development in Aerospace GmbH, Rigiplatz 5, 8006 Zürich, Switzerland

${ }^{4}$ Observatori de l'Ebre, CSIC - Universitat Ramon Llull, 43520 Roquetes, Spain

${ }^{5}$ EuropeanSpaceAgencyESA ESTEC, Keplerlaan 1, 2201AZ Noordwijk, Netherlands

Received 15 February 2018 / Accepted 16 February 2018

The online version of the original article can be found under: J. Space Weather Space Clim. 8 (2018) A05, https://oi.org/ $10.1051 / \mathrm{swsc} / 2017047$

An error occurred in the article "Feasibility of precise navigation in high and low latitude regions under scintillation conditions" published in Volume 8 (2018) A05. The name of the last author Raul "Orus Perez" was incomplete (Raul Orus). The correct name is "Raul Orus Perez".

The publisher apologizes for this mistake.

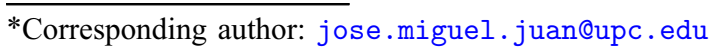

